\title{
Critical illness-related corticosteroid insufficiency: What we know and what we don't know
}

\author{
Ana M Rivas MD, David Sotello MD
}

\section{INTRODUCTION}

Glucocorticoids have an important role in the maintenance of vascular tone, endothelial integrity, and vascular permeability in the setting of acute illness. ${ }^{1}$ Elevation of plasma cortisol concentrations is part of the adaptive mechanisms in acute illness and inappropriately low plasma cortisol has been linked with increased mortality. ${ }^{2,3}$ Based on this, the concept of critical illness-related corticosteroid insufficiency $(\mathrm{CIRCl})$ was first introduced in $2008^{4}$ and refers to inadequate cellular corticosteroid activity for the severity of the patient's illness. ${ }^{4}$ This results in neurologic symptoms, such as confusion, delirium and coma, hypotension that is refractory to fluid resuscitation, decreased sensitivity to catecholamines, intolerance to enteral nutrition, hyponatremia, hypokalemia, hypoglycemia, and metabolic acidosis. ${ }^{5}$ To date, although the importance of glucocorticoids in the setting of acute illness is well accepted, the concept of $\mathrm{CIRCI}$, its diagnostic criteria, and appropriate treatment are not established, and in 2016, relative adrenal insufficiency was listed by Depuydt et al among "the ten diseases that are not diseases" in a publication in Intensive Care Medicine. $^{6}$

\section{Physiopathology of cortisol production AND METABOLISM IN ACUTE ILLNESS}

In the hypothalamic-pituitary-adrenal (HPI) axis, the "stress response" is initiated at the level of the paraventricular nucleus of the hypothalamus, which senses "stress" and in turn releases corticotropinreleasing hormone $(\mathrm{CRH})$ that activates the release

Corresponding author: Marcella Rivas Contact Information: Marcella.rivas@ttuhsc.edu DOI: 10.12746/swrccc.v7i31.595 of adrenocorticotropic hormone (ACTH) and subsequently stimulates cortisol synthesis and secretion by the adrenal gland. High cortisol levels in the setting of acute illness were initially thought to be mediated by an activation of the HPI axis through the above mechanism; however, a study comparing cortisol and ACTH levels in acutely ill intensive care unit (ICU) patients and healthy controls showed that critically ill patients actually have lower ACTH levels despite higher serum cortisol concentrations. ${ }^{7}$ Similarly, other studies have not found higher ACTH levels in acutely ill patients compared to controls. ${ }^{8}$ In addition, it has been shown that the secretion of cortisol in response to ACTH is unaltered during acute illness. ${ }^{9}$ In line with this, Boonen et al showed that cortisol production rate measured as "rate of cortisol appearance" in critical illness is increased to less than double compared to matched healthy individuals, not enough to explain higher cortisol concentration in blood seen in acute illness. On the other hand, plasma clearance of cortisol was decreased, as was the enzyme activity of 11B-hydroxysteroid dehydrogenase 1 and 5B-reductase in hepatic and adipose tissue, respectively, which are the enzymes that metabolize cortisol to its inactive form. ${ }^{7}$ The latter has now become the most accepted mechanism of cortisol increases in acute illness, but the reason why some patients do not have the capacity to respond to stress as effectively as others remains unexplained. Bile acids, which substantially increase during critical illness, have the ability to inhibit the enzymes that mediate this conversion. ${ }^{10}$ Better understanding of inhibitors of such enzymes could help determine factors that might contribute to our understanding of the development of $\mathrm{CIRCl}$.

\section{Diagnostic MOdalities of CIRCI}

There are no established diagnostic criteria for $\mathrm{CIRCl}$; proposed diagnostic criteria include a random 
cortisol level of $<10 \mu \mathrm{g} / \mathrm{dL}$ or an abnormal response to cosyntropin stimulation test, indicated by a delta cortisol of $<9 \mu \mathrm{g} / \mathrm{dL}$ after the administration of $250 \mu \mathrm{g}$ of cosyntropin. ${ }^{5}$ The most recent guidelines by the Society of Critical Care Medicine and European Society of Intensive Care Medicine make no recommendations about which test is superior. ${ }^{5}$ In the setting of septic shock, the Surviving Sepsis Guidelines recommend against using any of the above mentioned tests to determine if steroids should be used for the treatment of septic shock, and it is recommended that high dose hydrocortisone be used for shock that is refractory to fluids and moderate to high dose vasopressors without prior laboratory testing. ${ }^{11}$

The use of the cosyntropin stimulation test for the diagnosis of $\mathrm{CIRCI}$ is based on the results of a study by Annane et al in 189 patients with septic shock in which a lower mortality was observed in those with a delta cortisol of $>9 \mu \mathrm{g} / \mathrm{dL} .{ }^{12}$ In a subsequent study in which hydrocortisone and fludrocortisone were used in patients with septic shock, improved survival was observed in patients with an abnormal cosyntropin stimulation test done prior to the initiation of treatment. ${ }^{13} \mathrm{~A}$ criticism of this study is that $30 \%$ of the patients who were categorized as "non-responders" based on their responses to cosyntropin stimulation tests had received etomidate within 8 hours of testing. Considering the inhibitory effect of etomidate on cortisol synthesis for at least 24 hours,${ }^{14}$ the results of the study in relation to $\mathrm{CIRCI}$ or as proof of validity of the cosyntropin stimulation test for its diagnosis are questioned. The data were never reanalyzed after the exclusion of such patients..$^{15}$ Later studies have questioned the utility of a cosyntropin stimulation test for the diagnosis of $\mathrm{CIRCI}$ based on the rationale that a suboptimal response of cortisol to ACTH administration is actually the result of negative feedback from the excess cortisol that results from decreased cortisol clearance. ${ }^{16} \mathrm{~A}$ study by Loisa et al suggested that the result of the cosyntropin stimulation test in acutely ill patients with septic shock are inconsistent and not reproducible. These authors performed two consecutive cosyntropin stimulation tests in critically ill patients 24 hours apart. In septic shock no correlation was seen between the cortisol responses on day
1 and day 2. The majority of those patients who had poor cortisol responses on the first day demonstrated preserved adrenal function on the second day. In critically ill patients without septic shock, the results were more consistent. ${ }^{16}$

Finally, total cortisol, as is the case with total level of any other hormone, does not reflect the actual steroid effect, which is mostly mediated by free cortisol and its interaction with cortisol receptors at the tissue level. The affinity of cortisol binding globulin (CBG) for cortisol and the expression of cortisol receptors are altered in acute stress as an adaptation for survival. ${ }^{2}$ Because most of the circulating cortisol in human serum is protein-bound, changes in the binding proteins can alter measured serum total cortisol concentrations without necessarily influencing free concentrations of this hormone. Not uncommonly, critically ill patients present with low proteins, and when this is the case, the correlation of free and total cortisol has been described to be as low as $50 \%{ }^{5}$ In a study by Hamrahian et al, baseline serum total cortisol concentrations were found to be lower in patients with hypoproteinemia than in those with normal protein levels (defined as albumin $\leq 2.5$ and $>2.5 \mathrm{~g} / \mathrm{dL}$ respectively), while baseline serum free cortisol concentrations were similar in the two groups of patients. Cosyntropin-stimulated serum total cortisol concentrations were subnormal in 14 of the patients, all of whom had hypoproteinemia and had normal or high stimulated serum free cortisol concentrations. Acknowledging the limitations of total cortisol, some experts suggest that the cutoff for normal total cortisol levels should depend on the albumin levels that indirectly indicate the amount of protein available for cortisol binding. They suggest using a cutoff of $15 \mu \mathrm{g} / \mathrm{dL}$ when albumin levels are $>2.5 \mathrm{~g} / \mathrm{dL}$ and $10 \mu \mathrm{g} / \mathrm{dL}$ when albumin is $\leq 2.5 \mathrm{~g} / \mathrm{dL} .{ }^{15}$ Free cortisol levels could help better assess adrenal response to stress in acute illness, but normal values are not well defined. ${ }^{2}$ The processing of the sample is time consuming, and, therefore, the test is not widely available, making it an impractical tool in the setting of acute illness. When available, cutoff levels of $2.0 \mu \mathrm{g} / \mathrm{dL}$ have been recommended to identify patients who would benefit from steroid use. ${ }^{17}$ Formulas have been established to calculate free cortisol levels based on total cortisol 
levels, CBG and protein levels, but studies of the use of these formulas have shown that they are not precise and result in up to 66 percent error when compared to actual measured free cortisol. ${ }^{18}$

\section{THERAPEUTIC APPROACH OF CIRCI}

After the study mentioned above by Annane et $a l,{ }^{13}$ several subsequent studies have examined the benefits of steroids in sepsis and septic shock patients without finding reduced mortality with the use of steroids. ${ }^{19,20,21}$ In 2008 the "Corticosteroid Therapy of Septic Shock Study" (CORTICUS) group found no survival benefits in patients with septic shock treated with hydrocortisone $50 \mathrm{mg}$ every 6 hours, regardless of their responses to cosyntropin stimulation. ${ }^{19}$ Similarly, the "Hydrocortisone for Prevention of Septic Shock" (HYPRESS) study, in which patients with severe sepsis were assigned to receive a continuous infusion of $200 \mathrm{mg}$ of hydrocortisone for 5 days followed by dose tapering until day 11 or placebo, found no reduced occurrence of septic shock with the use of hydrocortisone. ${ }^{20}$ Finally, a study by Venkatesh et al that compared use of hydrocortisone for 7 days to placebo in septic shock patients also found no survival benefit with the use of steroids; reduction in time for shock resolution and ICU length of stay were the only benefits of steroids in this study. ${ }^{21}$ Two major differences between the above studies and the Annane study ${ }^{13}$ are that patients in the latter were more severely ill and received fludrocortisone along with hydrocortisone. A recent study in which a 7-day treatment with a 50-mg intravenous bolus of hydrocortisone every 6 hours and a daily dose of $50 \mu \mathrm{g}$ of oral fludrocortisone resulted in lower mortality at day 90 and ICU and hospital discharge than placebo among adults with septic shock. ${ }^{22}$ The Surviving Sepsis Guidelines recommend using hydrocortisone $200 \mathrm{mg}$ per day for shock refractory to fluid resuscitation and vasopressors and advise tapering the steroids once vasopressors are no longer needed. ${ }^{11}$

Finally, the benefits of using steroids in acute patients not in septic shock are less clear. In pneumonia there seems to be a mortality benefit in using steroids in cases of severe pneumonia when using hydrocortisone $200 \mathrm{mg}$ daily for five to seven days. In addition, steroids seem to reduce time to clinical stability and the length of hospital stay. ${ }^{23,24}$ Steroid benefit does not seem to correlate to the response to a cosyntropin stimulation test in this setting. ${ }^{25}$ The benefits of the use of hydrocortisone and fludrocortisone in patients with major trauma are not well established at this time. ${ }^{5}$ Relative adrenal insufficiency has also been described in patients with ischemic stroke ${ }^{26}$ and has been found to be highly prevalent in patients with liver disease, ${ }^{27}$ and burn patients. ${ }^{28}$ In these groups, patients with adrenal insufficiency are thought to have worse outcomes, ${ }^{29}$ but studies assessing treatment outcomes are lacking.

\section{Conclusion}

So, what do we know and what don't we know about the concept of $\mathrm{CIRCl}$ ? We certainly know that cortisol levels increase in the setting of acute illness as an adaptive response for better survival; this is achieved to a large extent through decreased metabolism of cortisol and to a lesser extent through increased cortisol production. We know that inability to maintain high cortisol levels in the setting of acute illness is associated with increased mortality in scenarios, like sepsis, septic shock, pneumonia, ischemic stroke, and liver disease among others. But we have not identified risk factors for $\mathrm{CIRCI}$ in patients who develop these conditions. We don't have clear diagnostic criteria that allow us to recognize those patients who fail to increase cortisol levels in acute illness; an assay to measure free cortisol that is readily available would possibly help us better assess cortisol status in acutely sick patients. We also know that when measuring serum total cortisol levels, albumin levels should be taken into consideration to account for the changes in bound cortisol when proteins are low. We know that patients with septic shock and severe pneumonia benefit from glucocorticosteroid treatment; in septic shock the benefits have been reported only when used with fludrocortisone.

Keywords: Adrenal insufficiency, cortisol, hydrocortisone 
Article citation: Rivas AM, Sotello D. Critical illnessrelated corticosteroid insufficiency: what we know and what we don't know. The Southwest Respiratory and Critical Care Chronicles 2019;7(31):44-48

From: Department of Internal Medicine, Texas Tech University Health Sciences Center, Lubbock, Texas

Submitted: $7 / 19 / 2019$

Accepted: 9/16/2019

Reviewer: Joaquin Lado MD, PhD

Conflicts of interest: none

This work is licensed under a Creative Commons

Attribution-ShareAlike 4.0 International License.

\section{REFERENCES}

1. Darlington DN, Kaship K, Keil LC, et al. Vascular responsiveness in adrenalectomized rats with corticosterone replacement. Am J Physiol 1989;256 (Heart Circ. Physiol. 25): H1274-81.

2. Boonen E, Von Den Berghe G. Cortisol metabolism in critical illness: implications for clinical care. Curr Opin Endocrinol Diabetes Obes 2014;21:185-192.

3. Widmer IE, Puder JJ, Konig C, et al. Cortisol response in relation to the severity of stress and illness. J Clin Endocrinol Metab 2005 Aug;90(8):4579-86.

4. Marik PE, Pastores SM, Annane D, et al. Recommendations for the diagnosis and management of corticosteroid insufficiency in critically ill adult patients: Consensus statements from an international task force by the American College of Critical Care Medicine. Crit Care Med 2008;36(6): 1937-49.

5. Annane D, Pastores SM, Rochwerg B, et al. Guidelines for the diagnosis and management of critical illness-related corticosteroid insufficiency (CIRCI) in critically ill patients (Part I): Society of Critical Care Medicine (SCCM) and European Society of Intensive Care Medicine (ESICM) 2017. Crit Care Med 2017;45(12):2078-88.

6. Depuydt PO, Kress JP, Salluh JIF. The ten "diseases" that are not true diseases. Intensive Care Med 2016 Mar;42(3):411-414.

7. Boonene E, Vervenne H, Meersseman P, et al. Reduced cortisol metabolism during critical illness. N Engl J Med 2013; 368:1477-88.

8. Michalaki M, Margeli T, Tsekouras A, et al. Hypothalamicpituitary-adrenal axis response to the severity of illness in non-critically ill patients: does relative corticosteroid insufficiency exist? Eur J Endocrinol 2010;162:341-347.
9. Peeters B, Boonen E, Langouche L, et al. The HPA axis response to critical illness: New study results with diagnostic and therapeutic implications. Molecular and Cellular Endocrinology 2015;408:235-240.

10. Vanwijngaerden YM, Wauters J, Langouche L, et al. Critical illness evokes elevated circulating bile acids related to altered hepatic transporter and nuclear receptor expression. Hepatology 2011;54(5):1741-52.

11. Rhodes A, Evans L, Alhazzanu W, et al. Surviving Sepsis Campaign: International Guidelines for Management of Sepsis and Septic Shock: 2016. Critical Care Med 2017;45(3): 486-552.

12. Annane D, Sebille V, Trouche G, et al. A 3-level prognostic classification in septic shock based on cortisol levels and cortisol response to corticotropin. JAMA 2000;283:1038-45.

13. Annane D, Sebille V, Charpentier C, et al. Effect of treatment with low dose of hydrocortisone and fludrocortisone on mortality in patients with septic shock. JAMA 2002;288: 862-871.

14. Thompson ML, Baker SN, Weant KA. Effects of etomidate on adrenal suppression: A review of intubated septic patients. Hosp Pharm 2014;49(2):177-83.

15. Hamrahian AH, Fleseriu M, AACE Adrenal Scientific Committee. Evaluation and management of adrenal insufficiency in critically ill patients: Disease state review. Endocr Pract 2017;23(6):716-25.

16. Loisa $P$, Uusaro A, Ruokonen E. A single adrenocorticotropic hormone stimulation test does not reveal adrenal insufficiency in septic shock. Anesth Analg 2005;101(6):1792-8.

17. Hamrahian AH, Oseni TS, Arafah BM. Measurements of serum free cortisol in critically ill patients. N Engl J Med 2004;350:1629-38.

18. Molenaar N, Groeneveld ABJ, De Jong MFC. Three calculations of free cortisol versus measured values in the critically ill. Clinical biochemistry 2015-7-15.

19. Sprung CL, Annane D, Keh D, et al. Hydrocortisone therapy for patients with septic shock. N Engl J Med 2008; 358:111-24.

20. Keh D, Trips E, Marx G, et al. Effect of hydrocortisone on development of shock among patients with severe sepsis. The HYPRESS Randomized Clinical Trial. JAMA. 2016; 316(17):1775-1785.

21. Venkatesh B, Finfer S, Cohen J, et al. Adjunctive glucocorticoid therapy in patients with septic shock. N Engl J Med 2018; 378:797-808.

22. Annane D, Renault A, Brun-Buisson C, et al. Hydrocortisone plus fludrocortisone for adults with septic shock. N Engl J Med 2018;378:809-18.

23. Annane D. Corticosteroids and pneumonia: time to change practice. The Lancet 2015;385:1484-85. 
24. Siemieniuk RAC, Meade MO, Alonso-Coello P, et al. Corticosteroid therapy for patients hospitalized with communityacquired pneumonia. Ann Intern Med 2015;163:519-528.

25. Blum CA, Schuetz P, Nigro N, et al. Cosyntropin testing does not predict response to glucocorticoids in communityacquired pneumonia in a randomized controlled trial. Clin Endocrinol (Oxf) 2018;1-9.

26. Wahab NA, Razak NZ, Sukor N, et al. Relative adrenal insufficiency amongst hospitalized mild to moderate acute ischemic stroke patients. Arch Iran Med 2015;18(2): 89-93.
27. O'Beirne J, Holmes M, Agarwal B, et al. Adrenal insufficiency in liver disease - what is the evidence? J Hepatol 2007; 47(3):418-23.

28. Mosier MJ, Lasinski AM, Gamelli RL. Suspected adrenal insufficiency in critically ill burned patients: etomidateinducedorcriticalillness-related corticosteroidinsufficiency?A Review of the Literature. J Burn Care Res. 2015;36(2): 272-8.

29. Graupera I, Pavel O, Hernandez-Gea V, et al. Relative adrenal insufficiency in severe acute variceal and non-variceal bleeding: influence on outcomes. Liver Int 2015;35:1964-73. 